\title{
Control in Power Electronics
}

\author{
Esam Alansari \\ Department of Electrical Engineering, University of Bridgeport, Bridgeport, CT, USA
}

\begin{abstract}
Metaheuristic methods have been demonstrated to be efficient tools to solve hard optimization problems. Most metaheuristics define a set of parameters that must be tuned. A good setup of that parameter values can lead to take advantage of the metaheuristic capabilities to solve the problem at hand. or predictive control algorithms based on typical linear controllers, exact and approximate methods for calculating optimal tunings are put forward. Optimally tuned predictive algorithms. an overview of results and future challenges related to temperature control and cost optimization in building energy systems. Control and economic optimization issues are discussed and illustrated through simulation examples. The paper concludes with results from model predictive control solutions and identification of important directions for future work.
\end{abstract}

Keywords: Control, Electronics, HVAC, Predictive.

\section{INTRODUCTION}

Predictive control is a very wide class of controllers that As with any generic process control problem, the objective have found rather recent application in the control of is to satisfy the output requirements in the most efficient power converters. Research on this topic has been way and hence with the least amount of input (energy). increased in the last years due to the possibilities of today's Because energy is a cost, control in buildings, as in most microprocessors used for the control. This paper presents other applications, can be translated to an economic the application of different predictive control methods to optimization problem. In simple mathematical terms, the power electronics and drives. A simple classification of problem can be stated as the minimization of the integral the most important types of predictive control is of the energy usage subject to constraints on the measured introduced, and each one of them is explained including variables (see Section 2 for an example). The real problem, some application examples. Predictive control presents however, is more complex because the constraints are not several advantages that make it suitable for the control of linear and the true goal is to minimize the energy cost (not power converters and drives. The different control just the energy usage), which may be nonlinear and time schemes and applications presented in this paper illustrate varying.

the effectiveness and flexibility of predictive control. Most electronic systems are subjected to a complex combination of internal and external transient thermal loads. The situation becomes even more conspicuous when the device experiences a sudden surge of power density, which can have detrimental effects on its components. In addition to this certain aspects associated with contemporary electronics such as nano/micro scale developments and interfacial resistance further intensify the problem of heat dissipation. All these factors pose serious challenges in the design of thermal control (TC) systems with capability to keep device operating temperatures under safe limits for their efficient and sustained working [1].

To meet the requirement on the safe operation area of the device, it has been proposed in literature to reduce the thermal resistance of the device by phase change cooling. The need for control in buildings usually resides in the mechanical and electrical systems that are installed to maintain a comfortable and safe indoor environment. A wide range of these systems can be found in buildings including heating, ventilating, air-conditioning (HVAC), lighting, security, elevators, escalators, fire detection and abatement. All these systems use energy and produce useful work as output. In the case of HVAC, energy is used to maintain temperature, humidity, and air quality at levels in accordance with the building purpose.

\section{PREDICTIVE CONTROL METHODS}

There are many scheduling problems in buildings that involve determining when to turn on and off equipment to satisfy load demands. Time-based scheduling is common where algorithms are used to determine when to turn on a device in order to reach desired conditions by a certain time. A specific example of this is "optimal start", which is used to turn on heating or cooling devices in buildings so that temperatures in zones are at setpoints when the occupancy time is reached (Yang, Yeo, \& Kim, 2003). Other types of scheduling problems involve determining how to operate multiple devices in series or in parallel. Devices that are in series are most commonly handled using split range control or finite state machines (Salsbury \& Chen, 2002). An example is moving from heating to cooling by splitting the output of a PID controller so that it maps on to two device inputs. Examples of parallel systems would be equivalent devices that serve different parts of a building. For example, a building might have multiple air-ventilation systems for different sections of a building. Coordination of these systems is rare, but algorithms are sometimes deployed to take account of start-up and shut-down events in order to minimize load spikes. The main characteristic of predictive control is the use of the model of the system for the prediction of the 
future behavior of the controlled variables. This the peak load period, while keeping thermal comfort as the information is used by the controller in order to obtain the ultimate goal. This strategy is known as pre-cooling. optimal actuation, according to a predefined optimization criterion [2].

The optimization criterion in the hysteresis-based predictive control is to keep the controlled variable within the boundaries of a hysteresis area, while in the trajectory based, the variables are forced to follow a predefined trajectory. In deadbeat control, the optimal actuation is the one that makes the error equal to zero in the next sampling instant. A more flexible criterion is used in MPC, expressed as a cost function to be minimized.

The difference between these groups of controllers is that deadbeat control and MPC with continuous control set need a modulator, in order to generate the required voltage. This will result in having a fixed switching frequency. The other controllers directly generate the switching signals for the converter, do not need a modulator, and present a variable switching frequency.

\section{LOAD SHIFTING AND DEMAND REDUCTION}

In the United States about $70 \%$ of electricity is consumed in commercial and residential buildings. To make it worse, the peak demands of building cooling (or in some regions heating) usually occur around the same time period during the day, making the electricity consumption at the peak time (known as demand) extremely high relative to the average consumption level. The high peak demand dictates that the power generation capacity has to be at least equal to the peak demand, or a blackout would occur. The issues associated with concentrated high demand are twofold. One is that new power plants have to be built to meet the increased demand. The other is that during offpeak hours many power plants have to be shut down or operated at a reduced load, sacrificing power generation efficiency and overall equipment utilization. The situation is worsened by the integration of renewable wind energy, which generates more during night, which is the off-peak consumption time. If the peak demand can be reduced by properly making use of storage capacity or managing the consumption pattern to be more friendly to the power generation, new power plants need not to be built and the efficiency of existing power plants is improved. Therefore, there is great interest to reduce the peak demand by shifting part of the peak load away from the peak time.

If large scale electricity storage were available at a feasible cost, the issue of load shifting would be resolved by implementing electrical storage. However, electricity storage is not currently viable due to the high cost and limited scales. Since buildings account for the majority of the electricity consumption and the peak load is usually caused by building cooling which is a thermal consumption, it is possible to use the building thermal storage capacity available in the buildings' thermal mass to shift the peak load to the off-peak period, usually before

The trajectories of the current vector for each possible switching state are computed, and predictions are made of the respective time intervals required to reach the error boundary again. These events also depend on the location of the error boundary, which is considered moving in the complex plane as commanded by the predicted current reference. The movement is indicated by the dotted circle in Fig. 4. The predictions of the switching instants are based on mathematical equations of the machine. The switching state vector that produces the maximum on-time is finally selected. This corresponds to minimizing the switching frequency.

The maximum possible switching frequency is limited by the computing time of the algorithms which determine the optimal switching state vector. Higher frequencies can be handled by employing the double prediction method: Well before the boundary is reached, the actual current trajectory is predicted in order to identify the time instant at which the boundary transition is likely to occur. The back electromotive force (EMF) vector at this time instant is predicted then. It is used for the optimal selection of the future switching state vector using the earlier described procedure. The attainable quality of control processes is to a considerable extent limited by inertness of a plant under control. Predictive algorithms based on typical linear algorithms of controllers (P, PI, PID, etc.) were proposed in $[1,2]$ in order to improve control quality of inert plants. Predictive algorithms implement the idea of partial or full compensation of the plant's inertia due to the fact that control is performed not from the plant's current state, but from its state expected after the prediction time $\tau$ ).

In this case, the regulator produces an anticipatory action $\mu(t)$, on the plant, due to which it becomes possible to partially compensate the plant inertia, i.e., the lagging response of the plant to actions of the control device. Pr $y(t+\tau$ pr A predictive controller (Fig. 1) consists of two parts: a controller itself with a typical control law $\mathrm{W}$ and a pre switched prediction element Pr with transfer function W (p) or with some nonlinear function $f(y, t)$. The prediction element, in combination with a setup unit, transforms the difference between the given $\mathrm{s}(\mathrm{t}) \mathrm{pr}$ and the actual values of a controlled quantity $y(t)$ into a signal of the control error $\varepsilon)$, expected after time $\tau \operatorname{pr} \operatorname{pr}(\mathrm{t})=\mathrm{s}(\mathrm{t})-$ $y(t+\tau$, according to which the control response $\mu(t)$ is formed in accordance with the adopted typical law. The derivative mode is often omitted in PID control strategies because it proves difficult to arrive by trial-and-error at a set of constants which meet plant requirements. The primary objective of this paper was to evaluate several model-based PID tuning methods. For lag-dominant processes, it was recommended that the SIMC algorithm first be employed to determine whether satisfactory performance can be obtained with PI control. If it cannot, then derivative action should be introduced using the DS-d technique. For delay-dominant systems, IMC tuning is 
preferred. It was observed that when configured with the same derivative filter factor, the series form of the PID controller produces smoother valve adjustments than the parallel version, at the expense of a slight decrease in best achievable performance. Increasing this parameter improves the control effort but limits achievable performance. ost industrial mechanical systems can be described by Lagrangian equations of motion, and their controllers are of the conventional PID type. Under some conditions for PID gains, the globally (or semi-globally) asymptotic stability of a PD/PID set-point regulation controller for a robot system without consideration of external disturbances was proved in[2]. Although the PID controller has been shown in practice to be effective when applied to a robot manipulator, an asymptotic stability [3].

In DMC, the initial costing horizon $\mathrm{Ni}$, the final costing horizon $\mathrm{Nr}$, the control horizon $\mathrm{N}$. and the move suppression factor $\mathrm{L}$ can be considered as the main parameters to be tuned and the output weighting polynomial $\mathrm{P}$ is taken to be unity. In GPC, in addition to the above parameters, $\mathrm{P}$ should also be. selected along with the model order, initial covariance and filter parameters. The default settings for DMC, referred to as $\mathrm{DMCl}$ in this study, are chosen as $\mathrm{Ni}=1, \mathrm{~N} 2=10, \mathrm{~N} .=1$ similar to the guidelines provided by Clarke et al. (1987) for GPC. Georgiou et al. (1988) and Maurath et al. (1985) suggested a different set oftuning guidelines as shown in Table 1, referred to as DMC2 and DMC3, respectively. Recently, Hinde and Cooper (1994) have proposed a pattern-based approach to excitation for process. For constructing the composite samples all the $\mathrm{C} / \mathrm{C}$ composite and plexiglass plates were first cut and machined to the required size. The $\mathrm{C} / \mathrm{C}$ sheets for casing were cut in a manner such that the sheet planes were oriented in a vertical direction having high value of thermal conductivity. A high thermal conductivity adhesive OMEGA bond 600 was used for gluing purpose. The casing plates were glued together in a stepwise manner allowing a curing time of $24 \mathrm{~h}$ after each step. The partition plates were first glued to the two plexiglass plates, followed by the top and bottom $\mathrm{C} / \mathrm{C}$ plates and one of the side $\mathrm{C} / \mathrm{C}$ plate. The casing thus prepared had one open side to fill the compartments with wax material. The required waxes were heated above their melting temperatures and slowly poured into the compartments such that approximately $85 \%$ volume of each compartment was filled with wax after it solidified. A volume of $15 \%$ for each compartment was kept empty to account for the wax thermal expansion upon melting. Finally, the second side $\mathrm{C} / \mathrm{C}$ plate was glued to complete the TC composite sample preparation [4].

\section{CONCLUSION}

An MPC strategy was used that directly handled inherent interactions in the system enabling improved set point tracking and disturbance rejection. The strategy also included economic optimization whereby the compressor speed was used as a proxy for energy use. Important system constraints were also integrated in the control strategy rather than being implemented separately as is the case in most current systems. The control objective was to satisfy set point tracking requirements within the constraint bounded region of operation and at the same time minimize the energy used by the compressor. Results showed significant improvements over the current state of the art. is important to emphasize the importance of using tuning methods to estimate parameter values.

In this study we have compared the capabilities of four well-known tuning methods proposed in the literature. All these methods present advantages and disadvantages and the idea of this study is to propose guidelines to select the tuning method to use according to the user requirements

\section{REFERNCES}

[1]. Irwin, J. D. (2002). Control in power electronics: selected problems M. P. Kazmierkowski, R. Krishnan, \& F. Blaabjerg (Eds.). Academic press.

[2]. Cortés, P., Kazmierkowski, M. P., Kennel, R. M., Quevedo, D. E., \& Rodríguez, J. (2008). Predictive control in power electronics and drives. Industrial Electronics, IEEE Transactions on, 55(12), 43124324.

[3]. Rodriguez, J., Kazmierkowski, M. P., Espinoza, J. R., Zanchetta, P., Abu-Rub, H., Young, H. A., \& Rojas, C. A. (2013). State of the art of finite control set model predictive control in power electronics. Industrial Informatics, IEEE Transactions on, 9(2), 1003-1016.

[4]. Blaabjerg, F., Chen, Z., \& Kjaer, S. B. (2004). Power electronics as efficient interface in dispersed power generation systems. Power Electronics, IEEE Transactions on, 19(5), 1184-1194. 